\title{
ON HURWITZ NUMBERS AND HODGE INTEGRALS
}

\author{
Torsten Ekedahl ${ }^{\dagger}$, Sergei Lando ${ }^{\ddagger}$ \\ Michael Shapiro ${ }^{\Uparrow}$, AND Alek Vainshtein ${ }^{\S}$ \\ $\dagger$ Dept. of Math., University of Stockholm, \\ S-10691, Stockholm, teke@matematik.su.se \\ $\ddagger$ Math. College, Moscow Independent \\ University, Moscow, lando@lando.mccme.rssi.ru \\ I Dept. of Math., Royal Institute of Technology, \\ S-10044, Stockholm, mshapiro@math.kth.se \\ $\S$ Dept. of Math. and Dept. of Computer Science, \\ University of Haifa, 31905 Haifa, alek@mathcs2.haifa.ac.il
}

\begin{abstract}
In this paper we find an explicit formula for the number of topologically different ramified coverings $C \rightarrow \mathbf{C} P^{1}$ ( $C$ is a compact Riemann surface of genus $g$ ) with only one complicated branching point in terms of Hodge integrals over the moduli space of genus $g$ curves with marked points.
\end{abstract}

1. Introduction and main results. For a compact connected genus $g$ complex curve $C$, let $\lambda: C \rightarrow \mathbf{C} P^{1}$ be a meromorphic function. We treat this function as a ramified covering of the sphere. Two ramified coverings $\left(C_{1} ; \lambda_{1}\right),\left(C_{2} ; \lambda_{2}\right)$ are called topologically equivalent if there exists a homeomorphism $h: C_{1} \rightarrow C_{2}$ making the following diagram commutative:

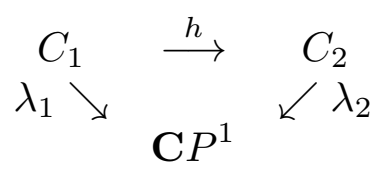

The critical values of topologically equivalent functions, i.e., the ramification points of the coverings, coincide, as do the genera of the covering curves. In his famous paper [11] Hurwitz initiated the topological classification of such coverings in the case when exactly one of the ramification points is degenerate, and the remaining points are nondegenerate. Below we refer to the degenerate ramification point as "infinity", and its preimages are called "poles". For a given set of orders $k_{1}, \ldots, k_{n}$ of $n$ distinct poles, the number of the equivalence classes of topologically nonequivalent ramified coverings with these orders of poles and prescribed nondegenerate ramification points is finite. This number $h_{g ; k_{1}, \ldots, k_{n}}$ (called the Hurwitz number) is independent of the exact location of the nondegenerate ramification points. The number of sheets in the covering is $k=k_{1}+\cdots+k_{n}$, and the space of all such coverings has dimension $d=k+n+2 g-2$ over C. Hurwitz posed the problem of finding $h_{g ; k_{1}, \ldots, k_{n}}$ explicitly. There exists a large 
number of publications on this topic written both by physicists and mathematicians, see e.g. $[1-3,8-11,15,16]$. Our aim is to express Hurwitz numbers in terms of intersection numbers for the Chern classes of certain line bundles on the moduli space of complex curves with $n$ marked points.

Let $\overline{\mathcal{M}}_{g ; n}$ denote the Deligne-Mumford compactification of the moduli space $\mathcal{M}_{g ; n}$ of genus $g$ curves with $n$ marked points, and let $\mathcal{L}_{i}$ be the line bundle on $\overline{\mathcal{M}}_{g ; n}$ whose fiber above a point $\left(C ; z_{1}, \ldots, z_{n}\right) \in \overline{\mathcal{M}}_{g ; n}$ coincides with the cotangent space to $C$ at $z_{i}$. The first Chern class of such a bundle is denoted by $c_{1}\left(\mathcal{L}_{i}\right)$.

The main result of the present paper is as follows. Let \# Aut $\left(k_{1}, \ldots, k_{n}\right)$ denote the number of automorphisms of the $n$-tuple $\left(k_{1}, \ldots, k_{n}\right), c\left(\Lambda_{g ; n}\right)$ denote the total Chern class of the relative dualizing sheaf over $\overline{\mathcal{M}}_{g ; n}$.

Theorem 1.1. The Hurwitz number $h_{g ; k_{1}, \ldots, k_{n}}$ equals

$$
\frac{d !}{\# \operatorname{Aut}\left(k_{1}, \ldots, k_{n}\right)} \prod_{i=1}^{n} \frac{k_{i}^{k_{i}}}{k_{i} !} \int_{\overline{\mathcal{M}}_{g ; n}} \frac{c\left(\Lambda_{g ; n}\right)}{\left(1-k_{1} c_{1}\left(\mathcal{L}_{1}\right)\right) \ldots\left(1-k_{n} c_{1}\left(\mathcal{L}_{n}\right)\right)}
$$

Here we admit as usual that the integral of a class with the degree different from that of the dimension of the manifold vanishes. A sketch of the proof is given in Section 2; a detailed account will appear elsewhere.

More general expressions $\int_{\overline{\mathcal{M}}_{g ; n}} c_{\alpha_{1}}\left(\Lambda_{g ; n}\right) \cdots c_{\alpha_{q}}\left(\Lambda_{g ; n}\right) c_{1}\left(\mathcal{L}_{1}\right)^{a_{1}} \cdots c_{1}\left(\mathcal{L}_{n}\right)^{a_{n}}$, including (1) as a special case, are called Hodge integrals; they are studied extensively in connection with Gromov-Witten invariants, quantum cohomologies and enumerative geometry (see e.g. $[4,7,12-14,17]$ ).

Combining Theorem 1.1 with Theorem 6 of [16], we recover the following recent calculation by C. Faber and R. Pandharipande [5].

\section{Theorem 1.2.}

$$
1+\sum_{g=1}^{\infty} t^{2 g} \int_{\overline{\mathcal{M}}_{g ; 1}} \frac{k^{g}+k^{g-1} c_{1}\left(\Lambda_{g ; n}\right)+\cdots+c_{g}\left(\Lambda_{g ; n}\right)}{1-c_{1}\left(\mathcal{L}_{1}\right)}=\left(\frac{t / 2}{\sin (t / 2)}\right)^{k+1} .
$$

The main ingredient of the proof of Theorem 1.1 is the following nonlinear version of the product formula for the total Segre classes of vector bundles. A similar homogeneous situation is considered in Chapter 5 of [6].

Let $\pi: \mathcal{A} \rightarrow X$ be a fiber bundle over a complex $m$-dimensional variety $X$ whose fibers are quasihomogeneous spaces $\mathbf{C}_{w_{0}, \ldots, w_{r}}^{r+1}$ with weights $\left(w_{0}, \ldots, w_{r}\right)$. Assume for simplicity that all $w_{i}$ are positive integers. We call $\mathcal{A}$ a bundle of quasihomogeneous spaces if the transition functions (possibly not linear) preserve the weights. Clearly, any vector bundle is a bundle of quasihomogeneous spaces with weights $(1, \ldots, 1)$. Any subbundle $\mathcal{Q} \subset \mathcal{A}$ whose fibers are quasihomogeneous cones (with the same weights $\left.\left(w_{0}, \ldots, w_{r}\right)\right)$ in $\mathbf{C}_{w_{0}, \ldots, w_{r}}^{r+1}$ is called a bundle of quasihomogeneous cones. Note that $\mathbf{C}^{*}$ acts fiberwise on both $\mathcal{A}$ and $\mathcal{Q}$ by multiplication of the corresponding coordinates by $t^{w_{i}}$. The quotient of $\mathcal{A}$ by the $\mathbf{C}^{*}$-action is fibered over $X$, and the fibers are isomorphic to the weighted projective spaces $P_{w_{0}, \ldots, w_{r}}$. We denote this quotient by $P \mathcal{A}$ and say that $p: P \mathcal{A} \rightarrow X$ is a bundle of weighted projective spaces. The quotient of $\mathcal{Q}$ by the $\mathbf{C}^{*}$-action is denoted by $P \mathcal{Q}$. Note that the fibers of both $P \mathcal{A}$ and $P \mathcal{Q}$ can be singular. 
Put $N=\operatorname{LCM}\left(w_{0}, \ldots, w_{r}\right)$. Denote by $\mathcal{Q} \oplus 1$ the fiberwise direct product of $\mathcal{Q}$ with the trivial one-dimensional vector bundle. Clearly, $\mathcal{Q} \oplus 1$ is also a bundle of quasihomogeneous cones with respect to the natural embedding $\mathcal{Q} \oplus 1 \subset \mathcal{A} \oplus 1$. The fibers of $\mathcal{A} \oplus 1$ are just $\mathbf{C}_{1, w_{0}, \ldots, w_{r}}^{r+2}$ (the weight of the new coordinate equals 1 ). By $q$ we denote the natural projection $q: \mathcal{Q} \oplus 1 \rightarrow X$. There is a natural sheaf $\mathcal{O}(N)$ of quasihomogeneous functions of quasihomogeneous degree $N$ on $P(\mathcal{A} \oplus 1)$ (which restricts also naturally to $P(\mathcal{A} \oplus 1))$.

Following [6] we define the total Segre class $s(\mathcal{Q}) \in H^{*}(X, \mathbf{Q})$ by the formula

$$
s(\mathcal{Q})=\sum_{i \geq 0} q_{*}\left(\frac{c_{1}(\mathcal{O}(N))^{i} \cap[P(\mathcal{Q} \oplus 1)]}{N^{i}}\right) .
$$

Assume that we are given a quasihomogeneous morphism $\psi: \mathcal{A} \rightarrow \mathcal{V}$ from a bundle of quasihomogeneous spaces $\mathcal{A}$ into a vector bundle $\mathcal{V}$ which is fiberwise of quasihomogeneous degree 1 . Consider a nonlinear exact triple

$$
0 \rightarrow \mathcal{Q}_{\psi} \stackrel{i}{\hookrightarrow} \mathcal{A} \stackrel{\psi}{\rightarrow} \mathcal{V} \rightarrow 0
$$

where the bundle of quasihomogeneous cones $\mathcal{Q}_{\psi}$ is the inverse image of the zero section in $\mathcal{V}$.

Theorem 1.3. Under the above assumptions one has

$$
s(\mathcal{A})=s\left(\mathcal{Q}_{\psi}\right) \cdot s(\mathcal{V}) .
$$

The authors are sincerely grateful to V.I.Arnold who introduced the last three authors to this subject and kept us informed about his own research. We are indebted to B. Dubrovin, L. Ernström, C. Faber, Yu. Manin, S. Natanzon, R. Pandharipande, M. Rosellen and, especially, B. Shapiro, for many fruitful discussions.

2. A sketch of the proof of Theorem 1.1. The space of all (holomorphic equivalence classes of) generic meromorphic functions on genus $g$ curves with prescribed orders $\left(k_{1}, \ldots, k_{n}\right)$ of poles will be called the Hurwitz space and denoted by $H_{g ; k_{1}, \ldots, k_{n}}$. It carries a natural complex structure. Observe that $H_{g ; k_{1}, \ldots, k_{n}}$ is naturally fibered over $\mathcal{M}_{g ; n}$ : the projection sends poles to marked points. There is also a natural mapping $L L: H_{g ; k_{1}, \ldots, k_{n}} \rightarrow \mathrm{Pol}_{d}$ called the Lyashko-Looijenga map, where $\mathrm{Pol}_{d}$ is the space of monic polynomials in one variable of degree $d=k+n+2 g-2 ; L L$ associates to each meromorphic function the unordered set of its critical values or, equivalently, the polynomial vanishing exactly on this set.

There exists a simple relation between the degree of the Lyashko-Looijenga map and the corresponding Hurwitz number (cf. [8]).

\section{Proposition 2.1.}

$$
\frac{\operatorname{deg} L L}{h_{g ; k_{1}, \ldots, k_{n}}}=\# \operatorname{Aut}\left(k_{1}, \ldots, k_{n}\right) \prod_{i=1}^{n} k_{i} .
$$

The following statement plays a key role in our construction. 
Proposition 2.2. There exists a compactification $\mathcal{H}_{g ; k_{1}, \ldots, k_{n}}$ of $H_{g ; k_{1}, \cdots, k_{n}}$ which is a bundle over $\overline{\mathcal{M}}_{g ; n}$; moreover, the Lyashko-Looijenga map extends to $\mathcal{H}_{g ; k_{1}, \ldots, k_{n}}$.

It is now easy to express $\operatorname{deg} L L$ in terms of intersection numbers. Indeed, let $x^{d}+a_{1} x^{d-1}+\cdots+a_{d} \in \mathrm{Pol}_{d}$; we denote by $D_{i}$ the divisor in $\mathcal{H}_{g ; k_{1}, \ldots, k_{n}}$ given by the quasihomogeneous equation $\left\{a_{i}=\right.$ const $\left.\cdot a_{1}^{i}\right\}$. Then $\operatorname{deg} L L=w\left(k_{1}, \ldots, k_{n}\right) \operatorname{deg}\left(\left[D_{2}\right] \cap\right.$ $\left.\cdots \cap\left[D_{d}\right]\right)$, where $\left[D_{i}\right] \in A_{*} P \mathcal{H}_{g ; k_{1}, \ldots, k_{n}}$ and $w\left(k_{1}, \ldots, k_{n}\right)$ is defined by the quasihomogeneous weights in the image and the preimage. (As usual, given an algebraic variety $X$, we write $A_{*} X$ for the rational equivalence group with coefficients in $\mathbf{Q}$; see [6] for details.)

Put $\Delta=c_{1}(\mathcal{Q}(1)) \in A_{*} P \mathcal{H}_{g ; k_{1}, \ldots, k_{n}}$. One can easily show that $\left[D_{i}\right]=i \cdot \Delta$ in the Chow ring. Therefore, $\operatorname{deg} L L=d ! w\left(k_{1}, \ldots, k_{n}\right) \int_{P \mathcal{H}_{g ; k_{1}, \ldots, k_{n}}} \Delta^{d}$. By the def-

inition of the top Segre class we have $\int_{P \mathcal{H}_{g ; k_{1}, \ldots, k_{n}}} \Delta^{d}=\int_{\overline{\mathcal{M}}_{g ; n}} s_{t o p}\left(\mathcal{H}_{g ; k_{1}, \ldots, k_{n}}\right)=$ $\int \overline{\mathcal{M}}_{g ; n} s\left(\mathcal{H}_{g ; k_{1}, \ldots, k_{n}}\right)$. Thus $\operatorname{deg} L L=d ! w\left(k_{1}, \ldots, k_{n}\right) \int \overline{\mathcal{M}}_{g ; n} s\left(\mathcal{H}_{g ; k_{1}, \ldots, k_{n}}\right)$.

The quasihomogeneity factor $w\left(k_{1}, \ldots, k_{n}\right)$ is given by the following statement (cf. [1, 8]).

\section{Proposition 2.3.}

$$
w\left(k_{1}, \ldots, k_{n}\right)=\prod_{i=1}^{n} \frac{k_{i}^{k_{i}}}{\left(k_{i}-1\right) !} .
$$

Denote by $\mathcal{P} \mathcal{P}_{g ; k_{1}, \ldots, k_{n}} \rightarrow \mathcal{M}_{g ; n}$ the fiber bundle of principal parts of germs of Laurent polynomials of degrees $k_{1}, \ldots, k_{n}$ at the marked points $P_{1}, \ldots, P_{n}$ on genus $g$ surfaces.

Proposition 2.4. There exists a compactification $\overline{\mathcal{P P}}_{g ; k_{1}, \ldots, k_{n}}$ of $\mathcal{P} \mathcal{P}_{g ; k_{1}, \ldots, k_{n}}$ which is a bundle of quasihomogeneous spaces over $\overline{\mathcal{M}}_{g ; n}$; moreover, $\mathcal{H}_{g ; k_{1}, \ldots, k_{n}}$ is a subbundle of quasihomogeneous cones in $\mathcal{P} \mathcal{P}_{g ; k_{1}, \ldots, k_{n}}$.

We now define a mapping $f: \overline{\mathcal{P P}}_{g ; k_{1}, \ldots, k_{n}} \rightarrow \Lambda_{g ; n}$. Let $p=\left(p_{1}, \ldots, p_{n}\right) \in \overline{\mathcal{P P}}_{g ; k_{1}, \ldots, k_{n}}$ be a collection of principal parts of Laurent germs at $P_{1}, \ldots, P_{n}$ and $\omega$ be a holomorphic 1-form. We put $\langle f(p), \omega\rangle=\sum_{i=1}^{n} \operatorname{Res}_{P_{i}}\left(p_{i} \omega\right)$. Plainly, $\mathcal{H}_{g ; k_{1}, \ldots, k_{n}}$ is the inverse image of the zero section. Hence, it is included in the nonlinear exact triple

$$
0 \rightarrow \mathcal{H}_{g ; k_{1}, \ldots, k_{n}} \stackrel{i}{\hookrightarrow} \overline{\mathcal{P}}_{g ; k_{1}, \ldots, k_{n}} \stackrel{f}{\rightarrow} \Lambda_{g ; n} \rightarrow 0
$$

Thus, applying Theorem 1.3 one obtains $s\left(\mathcal{H}_{g ; k_{1}, \ldots, k_{n}}\right)=s\left(\overline{\mathcal{P P}}_{g ; k_{1}, \ldots, k_{n}}\right) / s\left(\Lambda_{g ; n}\right)$. Taking into account that $s\left(\Lambda_{g ; n}\right)=1 / c\left(\Lambda_{g ; n}\right)$, where $c$ stands for the total Chern class, we rewrite the above formula as $s\left(\mathcal{H}_{g ; k_{1}, \ldots, k_{n}}\right)=s\left(\overline{\mathcal{P}}_{g ; k_{1}, \ldots, k_{n}}\right) \cdot c\left(\Lambda_{g ; n}\right)$.

On the other hand, projecting a collection of principal parts onto the collection of its leading terms one gets another nonlinear exact triple:

$$
0 \rightarrow \mathcal{B} \rightarrow \overline{\mathcal{P P}}_{g ; k_{1}, \ldots, k_{n}} \rightarrow \bigoplus_{i}\left(\mathcal{L}_{i}^{*}\right)^{\otimes k_{i}} \rightarrow 0
$$

where $\mathcal{B}$ is a trivial bundle of quasihomogeneous spaces of nonleading terms and $\mathcal{L}_{i}$ are the linear bundles mentioned in the introduction. Applying Theorem 1.3 one more time we complete the proof of Theorem 1.1. 


\section{REFERENCES}

[1] V. I. Arnold, Topological Classification of Trigonometric Polynomials and Combinatorics of Graphs with an Equal Number of Vertices and Edges, Funct. Anal. and Appl. 30 (1996), 1-17.

[2] M. Crescimanno, W. Taylor, Large $N$ phases of chiral QCD 2 , Nuclear Phys. B, 437 (1995), 3-24.

[3] B. A. Dubrovin, Geometry of 2D Topological Field Theories (1997), preprint.

[4] C. Faber, Algorithms for computing intersection numbers on moduli spaces of curves, with an application to the class of the locus of Jacobians, alg-geom/9706006.

[5] C. Faber, R. Pandharipande, Hodge integrals and Gromov-Witten theory, alg-geom 9810173.

[6] W. Fulton, Intersection Theory, 2nd edition, Springer, 1998.

[7] W. Fulton, R. Pandharipande, Notes on stable maps and quantum cohomology, Algebraic geometry, Santa Cruz 1995, Amer. Math. Soc., Providence, RI, 1997, pp. 45-96.

[8] V. Goryunov, S. K. Lando, On enumeration of meromorphic functions on the line (1997), preprint.

[9] I. P. Goulden, D. M. Jackson, Transitive factorisation into transpositions, and holomorphic mappings on the sphere, Proc. AMS 125 (1997), 51-60.

[10] I. P. Goulden, D. M. Jackson, A. Vainshtein, The number of ramified coverings of the sphere by the torus and surfaces of higher genera (1998), preprint.

[11] A. Hurwitz, Über Riemannische Flächen mit gegebenen Verzweigungspunkten, Math. Ann. 39 (1897), 1-60.

[12] M. Kontsevich, Intersection theory on the moduli space of curves and the matrix Airy function, Comm. Math. Phys. 147 (1992), 1-23.

[13] M. Kontsevich, Enumeration of rational curves via torus action, The Moduli Space of Curves, R. Dijkgraaf a.o. eds., Birkhäuser, 1995, pp. 335-368.

[14] M. Kontsevich, Yu. I. Manin, Gromov-Witten classes, quantum cohomology, and enumerative geometry, Comm. Math. Phys 164 (1994), 525-562.

[15] A. D. Mednykh, Branched coverings of Riemann surfaces whose branch orders coincide with the multiplicity, Comm. in Algebra 18 (1990), 1517-1533.

[16] B. Shapiro, M. Shapiro and A. Vainshtein, Ramified coverings of $S^{2}$ with one degenerate branching point and enumeration of edge-ordered graphs, Adv. in Math. Sci., vol. 34, AMS, Providence, RI, 1997, pp. 219-228.

[17] R. Vakil, Enumerative geometry of plane curves of low genus, alg-geom/9803007. 